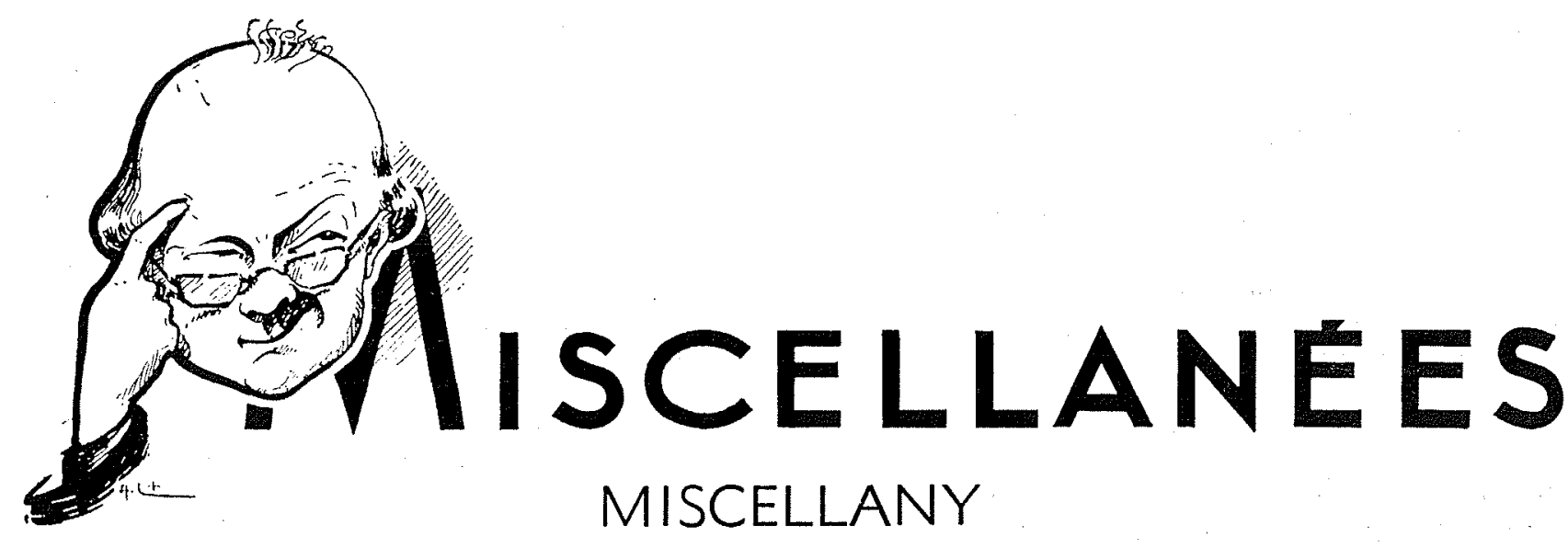

ayec la collaboration du Professeur Cyprien I.EBORGNe

\title{
OUILLE！HOULE！ HOULE！
}

(Problème $n^{0} 85$ )

\section{Mes chers amis,}

Beaucoup de gens prètendent que la meilleure saison pour profiter de la Côte d'Azur est l'arrièresaison, c'esț-à-dire le moment où j'écris ces lignes à votre intention... et à la leur. Car, puisque ces heureux bénéficiaires de vacances tardives sont au calme au bord de la Méditerranée, loin des foules, ils auront sans doute le loisir de se pencher sur le petit problème d'hydraulique maritime que le Sr. R. Alanaje vient de m'adresser et ils seront à

\section{LETTRE I}

Monsicur le Professeur,

Je veux que vous soyez le premier à l'apprendre: la houle n'existe pas et n'a jamais existé.

Notez que je m'en doutais depuis longtemps. Les marins professionnels n'ont jamais le mal de mer. Les marins occasionnels non plus, puisquion ne les a jamais vu rester couchés dans leurs cabines que par hygiène pour se reposer aprés les fatigues préparatoires du voyage, que par malchance pour soigner de brusques crises de foic compliquées de vomissements de bile imprévus, ou que par délicatesse pour ne pas obliger le commandant à se trouver tout seul à table avec eux.

Faut-il d'ailleurs vous dire que je n'ai jamais eu non plus le mal de mer puisque j'ai toujours pris la précaution de courir (ou de grimper) d'une position instantanée du métacentre du navire à l'autre pour m'éviter toute oscillation fatigante? Mais je m'aperçois aujourd'hui que j'avais bien tort de me démener autant puisque pied d'œuvre pour faire des études expérimentales très poussées sur l'existence ou l'inexistence de la houle. Aucun pêcheur ne leur refusera de sortir le «pointu», et, tout en taquinant la rascasse ou la girelle, ils seront à même de vérifier les dires de Airy, de Stokes ou de prêter une oreille complaisante à la théorie de mon correspondant, dont je vous livre ci-dessous la prose...

C. L.

la mer (que je n'avais pas le temps de regarder) ne bougeait pas pendant ce temps.

L'on s'explique d'ailleurs parfaitement la longue imposture de l'existence (prétendue) de la houle si l'on songe que c'est à des Anglais (Airy, Stokes, Rankine...) qu'on doit la plupart des études (sic) sur la question. Il était évidemment de l'intérêt de l'Angleterre de laisser croire qu'on était méchamment secoué sur mer pour pouvoir garder plus sûrement une maîtrise des mers qui fut son objectif pendant des siècles.

Adressons-nous donc à un neutre, en l'espèce Gerstner dont vous connaissez bien les calculs sur la question. Mais notez à ce propos qu'il n'y avait pas de mer en 'Tchécoslovaquie de son temps (pas plus d'ailleurs qu'aujourd'hui) et qu'il y a bien d'autres mathématiciens qui se sont illustrés, comme lui, dans l'étude d'éléments parfaitement imaginaires.

Les calculs de votre illustre prédécesseur ne. font appel qu'aux principes les plus indiscutables de la mécanique rationnelle et lon doit les tenir par conséquent pour absolument certains. Or chaque particule liquide décrit un cercle à 


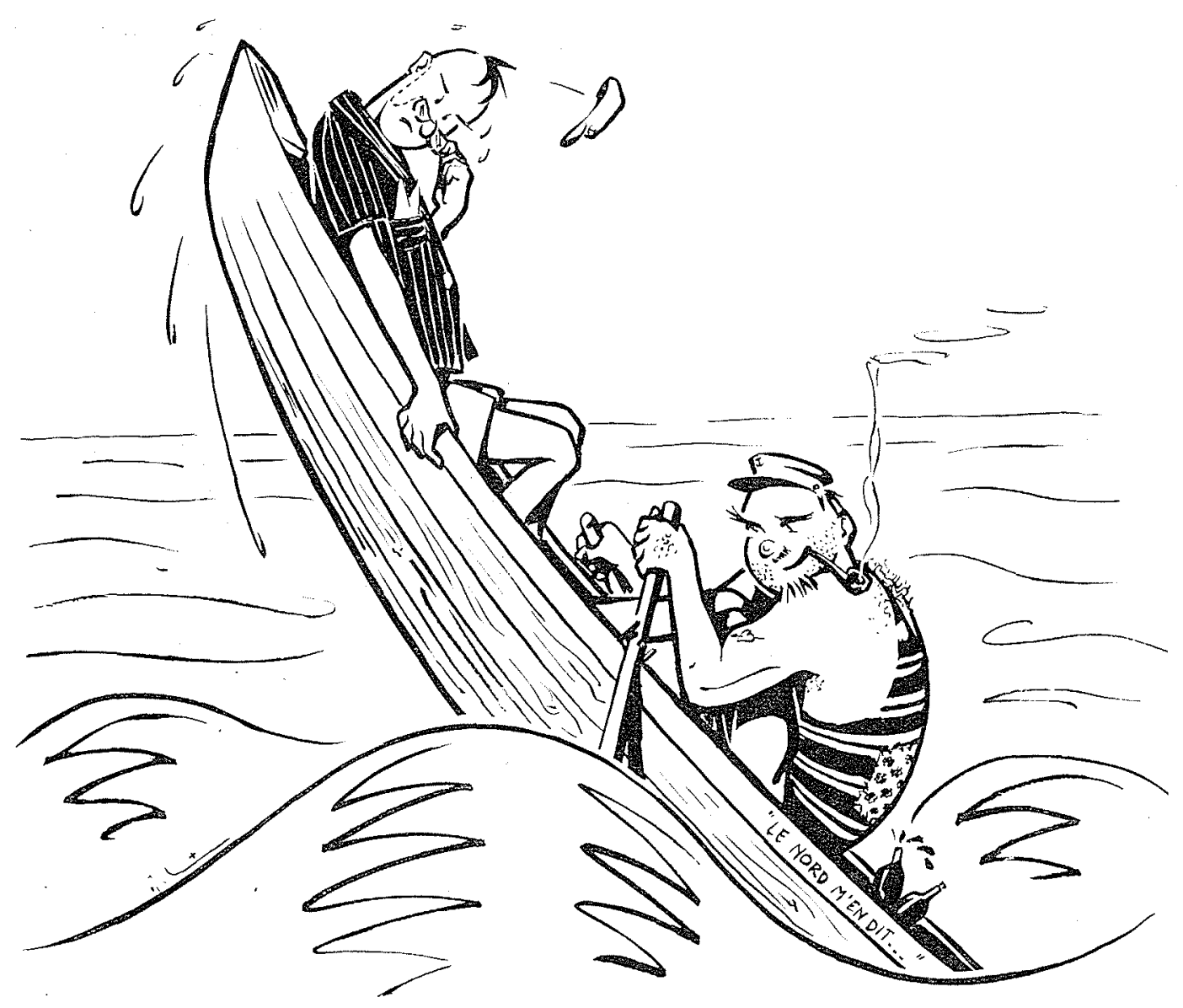

vitesse constante dans ce que Gerstner croyait être la houle. L'énergie cinétique de la particule est donc constante d'un bout à l'autre du mouvement et l'énergie de pression l'est forcément pour les particules de surface. Comme l'énergie totale reste par ailleurs constante, l'énergie potentielle des particules (qui constitue le solde) est elle-même constante et les particules de surface restent constamment à la même cote. La surface libre reste alors constamment horizontale et la mécanique rationnelle est bien là tout entière pour prouver que la houle n'existe pas.

En voici d'ailleurs une autre preuve, Monsieur le Professeur. Les particules liquides font toutes leur tour de cercle dans le même temps, et le rayon des cercles décroît avec la profondeur, la vitesse des particules est donc plus forte dans un sens que dans l'autre en tout point fixe du liquide puisqu'il s'agit de la vitesse vers le haut d'un cercle dans un cas et de la vitesse vers le

Maintenant il me faut ajouter autre chose. Le problème que je viens de vous poser, j'en soupconne l'auteur, cachant sous un pseudonyme trop transparent une personnalité dont l'autorité en matière de houle est bien connue.

J'en discutais justement il $y$ a quelques jours avec notre éminent et excellent ami, M. l'Ingénieur bas d'un cercle de centre plus élevé dans l'autre. Il doit done $y$ avoir un transport permanent de particules dans le sens où les vitesses sont plus grandes, alors que les particules restent comme vous le savez - moyennement fixes sur leurs cercles. Or c'est physiquement contradictoire. C'est done que la houle n'existe physiquement pas et cela vient, s'il en était encore besoin, à l'appui de la preuve déjà tirée des principes fondamentaux de la mécanique rationnelle.

Croyez, Monsieur le Professeur, à toute la joie que j'éprouve à vous faire bénéficier le premier d'une aussi précieuse découverte (dont les marchands de dramamine et la politique impériale britannique risquent seuls de souffrir). Et veuillez agréer, je vous prie, l'expression de mes sentiments de très haute considération.

\section{Sr. R. Alanaje.}

en chef Larras, auquel je ne saurais trop vous recommander de vous adresser si vous vous trouvez vraiment ébranlé dans vos convictions premières... D'autant plus que mon correspondant insiste, et découvre chaque jour de nouveaux et frappants arguments, comme en témoigne la missive $n^{\circ} 2$ que je vous transmets encore. 


\section{LETTRE II}

\section{Monsieur le Professeur,}

Je vous sais parfaitement convaincu comme moi de l'inexistence de la houle, dont je vous ai donné d'excellentes raisons l'autre jour.

Mais vous savez de votre côté quel est le poids de la tradition chez l'homme, et combien on trouve encore peu de gens capables de voir que la houle n'existe pas.

C'est donc pour vous aider à propager la bonne parole que je me permets de vous adresser aujourd'hui les deux nouvelles preuves que voici :

$1^{\circ}$ L'on raconte partout que la houle se propage avec une célérité $\sqrt{g H}$ en profondeur réduite $\mathrm{H}$.

L'on raconte également qu'elle se réfléchit sur une paroi verticale comme sur un miroir.

Toute houle attaquant une paroi verticale sous l'angle $\alpha$ devrait donc susciter des ondulations périodiques le. long de la paroi (1), et

(1) J'avais cru jadis tenir d'excellentes photos d'ondulations de cet ordre le long du parement extérieur de la jetée Butavand dans le port d'Alger. Mais vous savez les dangers du mirage, même pour les photographies, dans les déserts d'Afrique!. celles-ci devraient la parcourir à la vitesse $\sqrt{g \mathrm{H}}: \cos \alpha$.

Or, nous avons tous lu qu'aucune ondulation périodique ne peut se propager plus vite (ou moins vite) que $\sqrt{g \mathrm{H}}$, au premier ordre près, en profondeur $\mathbf{H}$.

Il y a donc là contradiction manifeste (surtout pour les houles pratiquement normales à la paroi) et c'est bien une fois de plus la preuve que la houle ne peut pas exister.

$2^{\circ}$ Nous avons tous lu que la houle double d'amplitude devant une paroi verticale, et cela quelle que soit l'incidence.

Elle devait donc doubler notamment d'amplitude sous l'incidence zéro.

Or, on prétend en même temps qu'elle se contente alors de courir tranquillement le long de la paroi sans s'y réfléchir. Et le plus grave c'est qu'on le démontre.

Voila donc une nouvelle contradiction manifeste et la preuve, une fois de plus, que la houle ne peut vraiment pas exister.

Veuillez agréer, Monsieur le Professeur, l'expression de mes sentiments de haute et respectueuse considération.

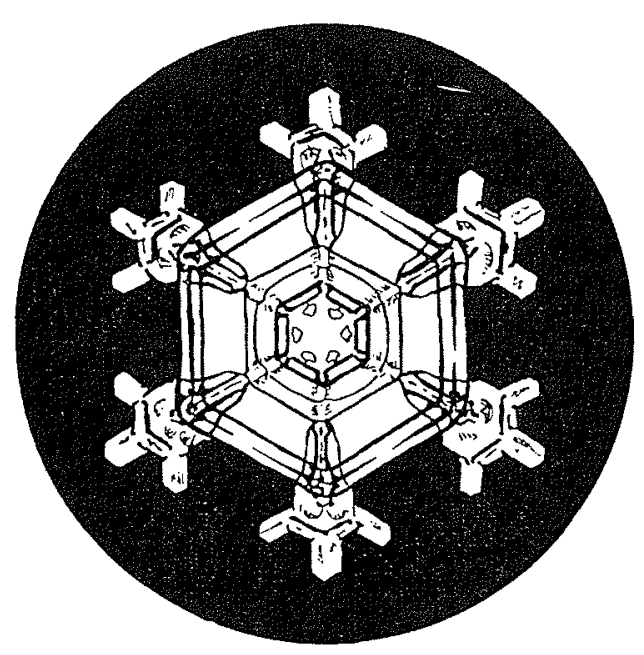

Sr. R. Alanaje. 